TITLE:

\title{
Evaluation of interlayer interfacial stiffness and layer wave velocity of multilayered structures by ultrasonic spectroscopy.
}

$\operatorname{AUTHOR}(\mathrm{S})$ :

Ishii, Yosuke; Biwa, Shiro

\section{CITATION:}

Ishii, Yosuke...[et al]. Evaluation of interlayer interfacial stiffness and layer wave velocity of multilayered structures by ultrasonic spectroscopy.. The Journal of the Acoustical Society of America 2014, 136(1): 183-191

ISSUE DATE:

2014-07

URL:

http://hdl.handle.net/2433/193601

\section{RIGHT:}

Copyright 2014 Acoustical Society of America. This article may be downloaded for personal use only. Any other use requires prior permission of the author and the Acoustical Society of America. 


\title{
Evaluation of interlayer interfacial stiffness and layer wave velocity of multilayered structures by ultrasonic spectroscopy
}

\author{
Yosuke Ishii and Shiro Biwa ${ }^{\text {a) }}$ \\ Department of Aeronautics and Astronautics, Graduate School of Engineering, Kyoto University, Katsura, \\ Nishikyo-ku, Kyoto 615-8540, Japan
}

(Received 10 January 2014; revised 1 May 2014; accepted 10 May 2014)

\begin{abstract}
An ultrasonic evaluation procedure for the interlayer interfacial normal stiffness and the intralayer longitudinal wave velocity of multilayered plate-like structures is proposed. Based on the characteristics of the amplitude reflection spectrum of ultrasonic wave at normal incidence to a layered structure with spring-type interlayer interfaces, it is shown that the interfacial normal stiffness and the longitudinal wave velocity in the layers can be simultaneously evaluated from the frequencies of local maxima and minima of the spectrum provided that all interfaces and layers have the same properties. The effectiveness of the proposed procedure is investigated from the perspective of the sensitivity of local extremal frequencies of the reflection spectrum. The feasibility of the proposed procedure is also investigated when the stiffness of each interface is subjected to small random fluctuations about a certain average value. The proposed procedure is applied to a 16-layered cross-ply carbon-fiber-reinforced composite laminate. The normal stiffness of resin-rich interfaces and the longitudinal wave velocity of plies in the thickness direction evaluated from the experimental reflection spectrum are shown to be consistent with simple theoretical estimations.

(C) 2014 Acoustical Society of America. [http://dx.doi.org/10.1121/1.4881920]
\end{abstract}

PACS number(s): 43.35.Zc, 43.40.Le, 43.35.Cg [GH]

Pages: 183-191

\section{INTRODUCTION}

With increasing application of multilayered structures in various technological fields, it has become a necessity to establish effective techniques of nondestructive evaluation for their property characterization and damage assessment. Since ultrasonic waves have a high potential for such a purpose, it is important to understand the wave propagation characteristics in multilayered structures. ${ }^{1,2}$ As a tool for the nondestructive inspection, mainly two types of ultrasonic features, the reflection/transmission characteristics of bulk waves or velocity/attenuation of guided waves, are usually considered, and a variety of theoretical approaches for such wave propagation behavior have been developed by many researchers. 3,4

It is well known that mechanical performance of layered structures can be critically degraded by interfacial imperfections between adjacent layers. Such imperfections include partial bonds, kissing bonds, thin adhesive layers, and so on. For example, in the case of fiber-reinforced composite laminates utilized widely in aerospace engineering, there exist resin-rich regions with a few microns thickness between each layer. For this reason, it is crucial to establish an ultrasonic evaluation method for the soundness of interlayer interfaces in order to ensure reliability as well as safety of layered structures.

When performing theoretical or numerical analysis of the wave propagation in a layered structure with imperfect interlayer interfaces, the wave interaction with these interfaces can be described by spring boundary conditions: ${ }^{5-11}$ The

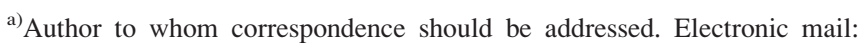
biwa@kuaero.kyoto-u.ac.jp
}

stresses are continuous while discontinuities are allowed in the displacements across the interface. The resulting displacement jumps are related to the stresses by the proportionality constants called the interfacial stiffnesses. The interfaces are therefore characterized by their normal and shear stiffnesses. In other words, the interfacial soundness can be evaluated by identifying these parameters.

Several procedures for the evaluation of interfacial stiffnesses have been proposed. For a single spring-type interface between two solids, the interfacial stiffnesses can be evaluated by the amplitude reflection or transmission coefficients of bulk ultrasonic waves, ${ }^{12-15}$ or the wave velocity and attenuation along the interface. ${ }^{16-18}$ Stiffness characterization of a spring-type joint interface of two plates by utilizing Lamb waves has been also studied recently. ${ }^{19}$ For double interfaces between a layer and two solids, Lavrentyev and Rokhlin ${ }^{20}$ proposed a technique to identify the interfacial stiffnesses from the ultrasonic reflection spectrum, and Singher et al $^{21}{ }^{21}$ studied the characteristics of guided waves propagating along the interfaces to evaluate the tangential interfacial stiffness. For layered structures, Leiderman et $a .^{22,23}$ proposed a technique to reconstruct the interfacial stiffness distribution due to localized adhesion flaws, and an ultrasonic characterization procedure to detect the interfacial weakness using the adaptive filtering method was also proposed by Jian et al. ${ }^{24}$ Recently, Ishii and Biwa ${ }^{25}$ proposed a stiffness evaluation procedure for a multilayered structure by using local extremal frequencies of the amplitude reflection spectrum, which is effective in obtaining a representative value of interfacial stiffness for the whole structure.

In many practical situations, not only the interfacial stiffness but also bulk properties of layers are often unknown; hence, it is desirable to evaluate them simultaneously. Baltazar 
et al $^{26}$ identified the bulk properties of an intermediate layer of a three-layered structure as well as their interfacial stiffnesses by the ultrasonic reflection spectra at two incident angles (normal and oblique). For the case of more general multilayered structures with imperfect interfaces, however, such evaluations have not been reported to the authors' knowledge.

In this paper, the above-mentioned procedure by Ishii and $\mathrm{Biwa}^{25}$ is extended to establish a new method to evaluate the layer properties, in particular the longitudinal wave velocity in the thickness direction (direction normal to the layers), as well as the interlayer interfacial normal stiffness of a multilayered plate-like structure by the ultrasonic spectroscopy. The proposed method aims at obtaining representative values for the layer wave velocity and the interfacial stiffness of a multilayered structure when all layers and interfaces have nearly equal properties.

This paper is structured as follows. In Sec. II, the theoretical expression of the reflection spectrum for a multilayered structure with spring-type interfaces derived in Ref. 25 is rewritten in terms of newly defined non-dimensional parameters, and the dependence of the reflection spectrum on these parameters is clarified. This results in the proposal of an identification procedure for the interlayer interfacial normal stiffness and the layer longitudinal wave velocity in the thickness direction by making use of the local extremal frequencies of the spectrum (Sec. III A). Some considerations are made in Sec. III B in regard to the feasibility and the effectiveness of the proposed procedure. As a demonstrative example, the ply longitudinal wave velocity in the thickness direction and the interfacial normal stiffness of a carbonfiber-reinforced composite laminate are evaluated from the experimental reflection spectrum in Sec. IV. The conclusion of this study is summarized in Sec. V.

\section{THEORETICAL BACKGROUND}

Consider a multilayered structure consisting of $N$ equal elastic layers of density $\rho$, longitudinal wave velocity $c$, and thickness $h$, with $(N-1)$ spring-type interlayer interfaces of the stiffness $K_{\mathrm{N}}$ as shown in Fig. 1. The structure is embedded between two semi-infinite media of density $\rho_{0}$ and longitudinal wave velocity $c_{0}$ with perfect acoustical coupling: both stresses and displacements are continuous across the boundaries at $x=X_{0}$ and $x=X_{N}$.

If the longitudinal harmonic wave with unit amplitude $\exp \left(i k_{0} x-i \omega t\right)$ impinges perpendicularly on the structure from

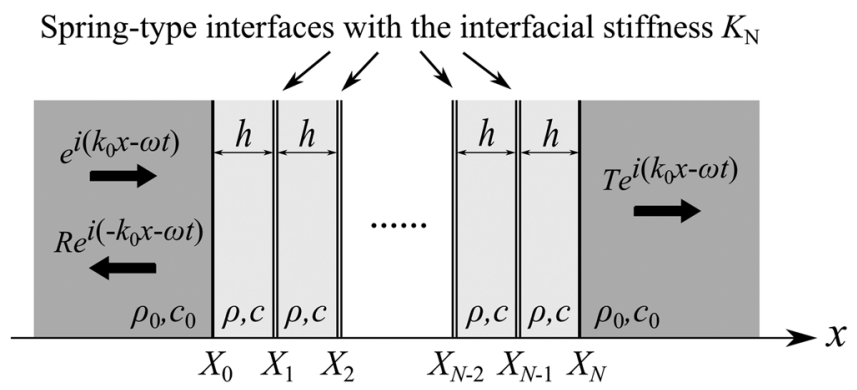

FIG. 1. An $N$-layered structure with spring-type interlayer interfaces embedded between two semi-infinite media.
$x<X_{0}$, the reflected wave $R \exp \left(-i k_{0} x-i \omega t\right)$ for $x<X_{0}$ and the transmitted wave $T \exp \left(i k_{0} x-i \omega t\right)$ for $x>X_{N}$ are generated (Fig. 1). Here $\omega(=2 \pi f)$ is the angular frequency, $k_{0}=\omega / c_{0}$ is the wave number in the semi-infinite media, and $R$ and $T$ are the reflection and transmission coefficients, respectively. In the previous study, ${ }^{25}$ the characteristics of the reflection coefficient $R$ were investigated theoretically by the transfer-matrix method ${ }^{27-30}$ combined with Bloch's theorem, ${ }^{31,32}$ and it was shown that when the frequency lies in the passbands of the corresponding infinite periodic structure, i.e., when the Bloch phase $\beta$ is real, $R$ can be written as

$$
\begin{aligned}
& R=\frac{L_{3}}{L_{1}+i L_{2}} \exp \left(2 i k_{0} X_{0}\right), \\
& L_{1}=2 \kappa f^{*} \sin \{(N-1) \beta\}+\left(\zeta^{2}+1\right) \sin (N \beta) \sin \left(\gamma f^{*}\right), \\
& L_{2}=2 \zeta\left[\sin (N \beta) \cos \left(\gamma f^{*}\right)-\sin \{(N-1) \beta\}\right] \\
& L_{3}=2 \kappa f^{*} \sin \{(N-1) \beta\}-\left(\zeta^{2}-1\right) \sin (N \beta) \sin \left(\gamma f^{*}\right),
\end{aligned}
$$

where non-dimensional interfacial compliance $\kappa$, nondimensional slowness in the layer $\gamma$, acoustic impedance ratio $\zeta$, non-dimensional frequency $f^{*}$, and the Bloch phase $\beta$ are respectively given by

$$
\begin{aligned}
& \kappa=\frac{\rho c \pi f_{0}}{K_{\mathrm{N}}}, \quad \gamma=\frac{2 \pi h f_{0}}{c}, \quad \zeta=\frac{\rho c}{\rho_{0} c_{0}}, \quad f^{*}=\frac{f}{f_{0}}, \\
& \cos \beta=\cos \left(\gamma f^{*}\right)-\kappa f^{*} \sin \left(\gamma f^{*}\right),
\end{aligned}
$$

where $f_{0}$ is an arbitrary normalization factor. In the passbands, $L_{3}$ in Eq. (1d) is real and can be written as

$$
L_{3}=\sqrt{A^{2}+B^{2}} \sin (N \beta+\varphi),
$$

where $A, B$, and $\varphi$ are given by

$$
\begin{aligned}
& A=2 \kappa f^{*} \cos \beta-\left(\zeta^{2}-1\right) \sin \left(\gamma f^{*}\right), \quad B=-2 \kappa f^{*} \sin \beta, \\
& \cos \varphi=\frac{A}{\sqrt{A^{2}+B^{2}}}, \quad \sin \varphi=\frac{B}{\sqrt{A^{2}+B^{2}}} .
\end{aligned}
$$

Equations (1) and (3) indicate that the reflection coefficient $R$ vanishes when

$$
N \beta+\varphi=m \pi,
$$

where $m$ is an integer. The frequencies of zeros of the reflection coefficient are hence determined by the nondimensional parameters $N, \kappa, \gamma$, and $\zeta$, and so is the number of these zeros in each passband.

Figure 2 shows the variation of the amplitude reflection coefficient $|R|$ with the non-dimensional frequency $f^{*}$ for different $\kappa, \gamma$, and $\zeta$ with a fixed $N=16$. The amplitude spectra of the corresponding infinite periodic 
(a) $\kappa=0.03, \gamma=0.3, \zeta=3$

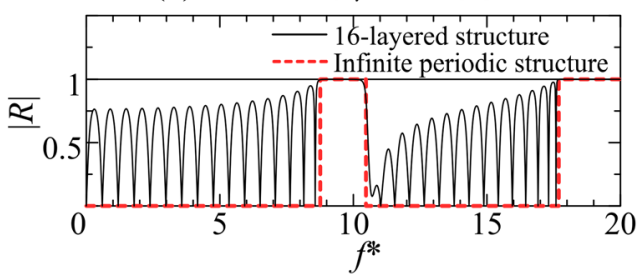

(b) $\kappa=0.06, \gamma=0.3, \zeta=3$

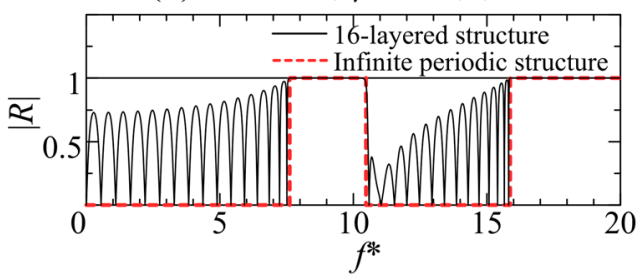

(c) $\kappa=0.06, \gamma=0.3, \zeta=2$

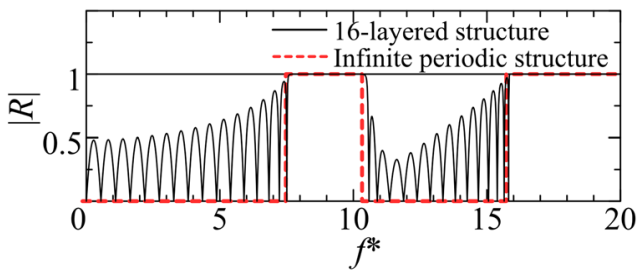

(d) $\kappa=0.06, \gamma=0.5, \zeta=2$

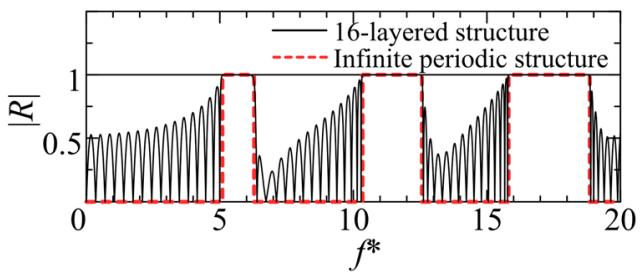

FIG. 2. (Color online) The amplitude reflection spectra of a multilayered structure for $N=16$, (a) $\kappa=0.03, \gamma=0.3$, and $\zeta=3$, (b) $\kappa=0.06, \gamma=0.3$, and $\zeta=3$, (c) $\kappa=0.06, \gamma=0.3$, and $\zeta=2$, and (d) $\kappa=0.06, \gamma=0.5$, and $\zeta=2$, together with the reflection spectrum of the corresponding infinite periodic structures.

structures are also shown in Fig. 2 for comparison, where $|R|$ becomes either zero (passbands) or unity (stop bands). Originally, the terms of pass and stop band can be defined for infinite periodic structures. In what follows, these terms are also used for finite structures; namely, the term pass (stop) band refers to the band of frequency which belongs to the pass (stop) band of the corresponding infinite periodic structure.

It is noted that the use of non-dimensional parameters in the formulation has an advantage that it enables us to study the influence of each parameter on the spectrum in a universal manner. It is found in Fig. 2 that the non-dimensional parameters $\kappa, \gamma$, and $\zeta$ have effects to change the width of the frequency range corresponding to the stop bands [compare Figs. 2(a) and 2(b)], to expand the spectrum with respect to the frequency axis [compare Figs. 2(c) and 2(d)], and to change the magnitude of $|R|$ in the passbands [compare Figs. 2(b) and 2(c)], respectively. It is also seen that as mentioned above, each spectrum in Fig. 2 has a certain number of zeros in each passband. This is governed by the number of layers $N$ as shown in the previous paper. ${ }^{25}$

\section{EVALUATION OF INTERLAYER INTERFACIAL STIFFNESS AND LAYER WAVE VELOCITY}

\section{A. Outline of the evaluation procedure}

In the preceding section, it has been shown that the amplitude reflection coefficient $|R|$ for a multilayered structure takes zeros at certain discrete frequencies determined by the number of layers $N$ and the non-dimensional parameters $\kappa, \gamma$, and $\zeta$. In other words, the latter three parameters can possibly be evaluated inversely by measuring these frequencies, provided that $N$ is known and that all layers and interfaces respectively have the same properties.

It is recognized from Fig. 2 that the amplitude reflection coefficient $|R|$ takes local maxima and minima (including zeros) in the passbands, whose frequencies $f_{\mathrm{e}}^{*}$ are also determined by $N, \kappa, \gamma$, and $\zeta$. These points are easier to obtain from the experimental reflection spectrum compared to the exact zeros. The variations of these points with $\kappa, \gamma$, and $\zeta$ for a fixed $N=16$ are shown in Figs. 3, 4, and 5, respectively. Clear dependence of the extremal frequencies on $\kappa$ (except in the small $\kappa$ range) and $\gamma$ can be observed, which will make the precise determination of $\kappa$ and $\gamma$ possible. On the other hand, it is seen in Fig. 5 that these frequencies are mostly insensitive to $\zeta$. This can be explained by the characteristic of $\zeta$ mentioned in the foregoing section; $\zeta$ has an effect to mainly change the magnitude of the reflection coefficient $|R|$ in the passbands. This weak dependence on $\zeta$ implies that it will be difficult to precisely evaluate $\zeta$ by the extremal frequencies.

Dimensional parameters of practical interest, i.e., the interlayer interfacial stiffness $K_{\mathrm{N}}$, the wave velocity in the layers $c$, and the density of the layers $\rho$, can be given in terms of the non-dimensional parameters $\kappa, \gamma$, and $\zeta$ as

$$
K_{\mathrm{N}}=\pi \rho_{0} c_{0} f_{0} \frac{\zeta}{\kappa}, \quad c=\frac{2 \pi h f_{0}}{\gamma}, \quad \rho=\frac{\rho_{0} c_{0}}{2 \pi h f_{0}} \gamma \zeta
$$

where the other dimensional parameters, the layer thickness $h$, the density $\rho_{0}$ and wave velocity $c_{0}$ of the semi-infinite

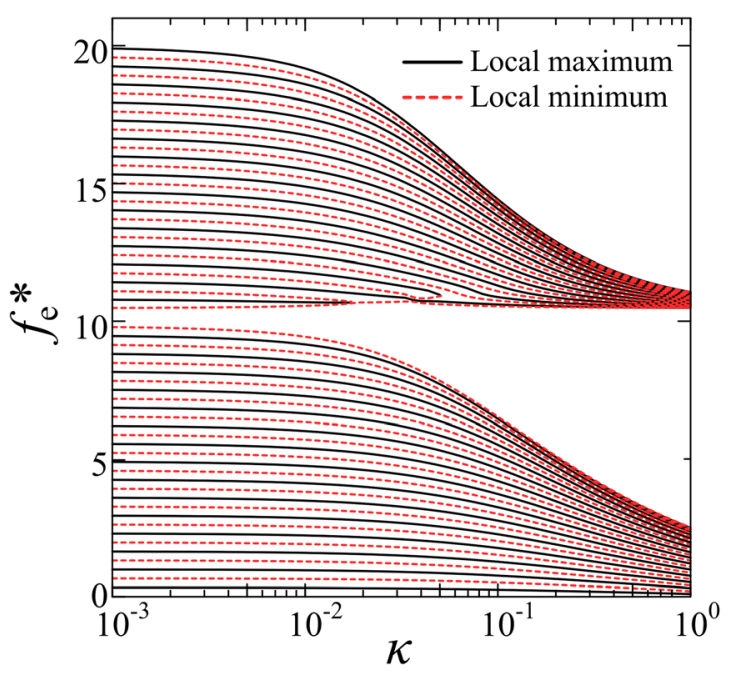

FIG. 3. (Color online) The extremal frequencies in the 1st and 2nd passbands of the amplitude reflection spectrum as a function of $\kappa$ with fixed $\gamma=0.3, \zeta=3$, and $N=16$. 


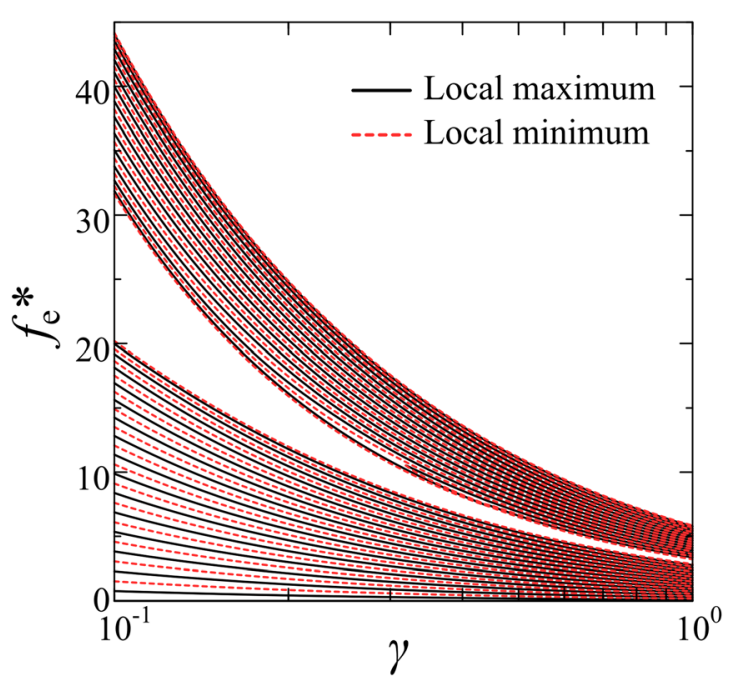

FIG. 4. (Color online) The extremal frequencies in the 1st and 2nd passbands of the amplitude reflection spectrum as a function of $\gamma$ with fixed $\kappa=0.03, \zeta=3$, and $N=16$.

media, and the normalization factor $f_{0}$, are assumed to be known in advance. As mentioned above, however, the precise determination of $\zeta$ will be difficult. In order to circumvent this problem, $\zeta$ is rewritten in terms of $\gamma$ and a newly introduced non-dimensional parameter $\chi$ as

$$
\zeta=\frac{\chi}{\gamma}, \quad \chi=\frac{2 \pi \rho h f_{0}}{\rho_{0} c_{0}} .
$$

Under the assumption that $\chi$ is known in advance, i.e., $\rho$ is also known in addition to $h, \rho_{0}, c_{0}$, and $f_{0}$, the sought-for parameters $K_{\mathrm{N}}$ and $c$ are given in terms of $\kappa$ and $\gamma$ as

$$
K_{\mathrm{N}}=\frac{\chi}{\kappa \gamma} \pi \rho_{0} c_{0} f_{0}, \quad c=\frac{2 \pi h f_{0}}{\gamma} .
$$

Since the number of dimensional parameters to be evaluated is reduced in comparison with Eq. (6), precise determination

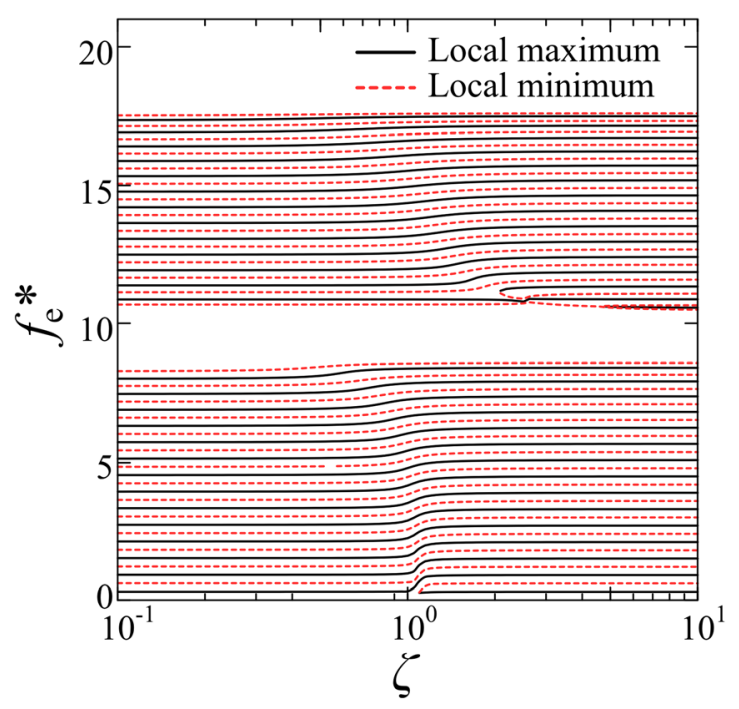

FIG. 5. (Color online) The extremal frequencies in the 1st and 2nd passbands of the amplitude reflection spectrum as a function of $\zeta$ with fixed $\kappa=0.03, \gamma=0.3$, and $N=16$. of $K_{\mathrm{N}}$ as well as $c$ is possible due to the absence of $\zeta$ in the equation.

The procedure to evaluate the interlayer interfacial stiffness $K_{\mathrm{N}}$ and the layer wave velocity $c$ is put forward as follows. The ultrasonic reflection spectrum for a multilayered structure is first measured experimentally with a broadband incident wave and the extremal frequencies $f_{i}^{\exp }(i=1,2, \ldots$, $m)$ are extracted from spectral analysis. The set of $\kappa$ and $\gamma$ is then identified as the optimal point which minimizes the evaluation function $J(\kappa, \gamma)$ defined as

$$
J(\kappa, \gamma)=\frac{1}{m} \sum_{i=1}^{m}\left[\frac{f_{i}^{\exp }-f_{i}^{\text {theor }}(\kappa, \gamma)}{f_{i}^{\exp }}\right]^{2},
$$

where $m$ is the number of extrema considered and $f_{i}^{\text {theor }}(\kappa, \gamma)$ are the theoretical extremal frequencies as functions of $\kappa$ and $\gamma$ with known $N$ and $\chi$, which are extracted from the numerically calculated spectrum by Eq. (1) with a sufficiently small frequency increment $\left(\Delta f^{*}=1 \times 10^{-4}\right)$. Finally, $K_{\mathrm{N}}$ and $c$ are obtained by Eq. (8) with the identified $\kappa$ and $\gamma$ and known $\chi$, $h, \rho_{0}, c_{0}$, and $f_{0}$.

\section{B. Feasibility of the proposed procedure}

\section{Effects of viscoelasticity of layers}

It should be remembered that the above discussions have been made with the assumption that each layer is elastic. In the case of layered structures made of polymeric materials, however, each layer has certain viscoelastic nature at ultrasonic frequencies. It is therefore imperative to examine if the proposed procedure is still feasible in the presence of the viscoelasticity.

In the previous study, ${ }^{25}$ the variation of the extremal frequencies of the reflection spectrum with the interfacial stiffness $K_{\mathrm{N}}$ was investigated for the cases where the layers are elastic and where the layers are viscoelastic. Since the procedure proposed in this study incorporates not only $K_{\mathrm{N}}$ but also the layer wave velocity $c$, the variations of the reflection spectrum with non-dimensional parameters $\kappa$ and $\gamma$ were examined in a similar fashion. As a result, it was found that local extremal frequencies in the first passband rarely depend on whether the layers are elastic or viscoelastic. On the other hand, as mentioned in Ref. 25, the disappearance of some extremal points due to the introduction of viscoelasticity was observed in the second passband at high $\kappa$ and $\gamma$ ranges; nevertheless the existing extremal frequencies still agreed well with those without viscoelasticity. Consequently, it has been concluded that the proposed evaluation procedure can be applied, even if the layers possess unknown viscoelastic nature.

\section{Number of extrema used for evaluation}

It is seen in Figs. 3 and 4 that the variations of the extremal frequencies of the amplitude reflection spectrum with $\kappa$ and $\gamma$ are both monotonic in the first passband, implying that a trade-off between $\kappa$ and $\gamma$ will occur if the extremal points only in the first passband are used for the evaluation. As an example, the amplitude reflection spectra 
for some sets of $\kappa$ and $\gamma$ with fixed $N=16$ and $\chi=0.84$ are shown in Fig. 6, where in spite of different values of $\kappa$ and $\gamma$, their extremal frequencies in the first passband are almost identical. It is hence necessary to use the extremal points in not only the first but also the second passband for unique determination of $\kappa$ and $\gamma$.

It should be noted that when calculating the evaluation function $J$ in Eq. (9), it is required to identify the theoretical extremal point $f_{i}^{\text {theor }}(\kappa, \gamma)$ corresponding to one measured by the experiment $f_{i}^{\text {exp }}$. It is seen in Figs. 3 and 4 that the number of extremal points in the first passband is constant regardless of $\kappa$ and $\gamma$, making the correspondence of $f_{i}^{\text {theor }}(\kappa, \gamma)$ to $f_{i}^{\text {exp }}$ straightforward. In contrast, such a number does change for the second passband due to the presence of branch-offs seen in Figs. 3 and 4. As a result, such correspondence is not clear for the extrema in the second passband; hence, a searching process of $f_{i}^{\text {theor }}(\kappa, \gamma)$ corresponding to $f_{i}^{\text {exp }}$ is required for each $\kappa$ and $\gamma$. In the examples shown below, all of their possible correspondences are taken into account and one of them is chosen so that the resulting evaluation function is minimized.

\section{Sensitivity of the proposed procedure}

To investigate the effectiveness of the proposed evaluation procedure, the sensitivity of the extremal frequencies of the amplitude reflection spectrum to the non-dimensional parameters is considered following the manner employed by Lavrentyev and Rokhlin. ${ }^{33}$ Regarding each extremal frequency of the reflection spectrum $f_{\mathrm{e}}^{*}$ as a function of a parameter $p(\kappa$ or $\gamma)$, the relative errors in $p$ and $f_{\mathrm{e}}{ }^{*}, \varepsilon_{p}=\delta p / p$ and $\varepsilon_{f_{\mathrm{e}}^{*}}=\delta f_{\mathrm{e}}^{*} / f_{\mathrm{e}}^{*}$, are related by
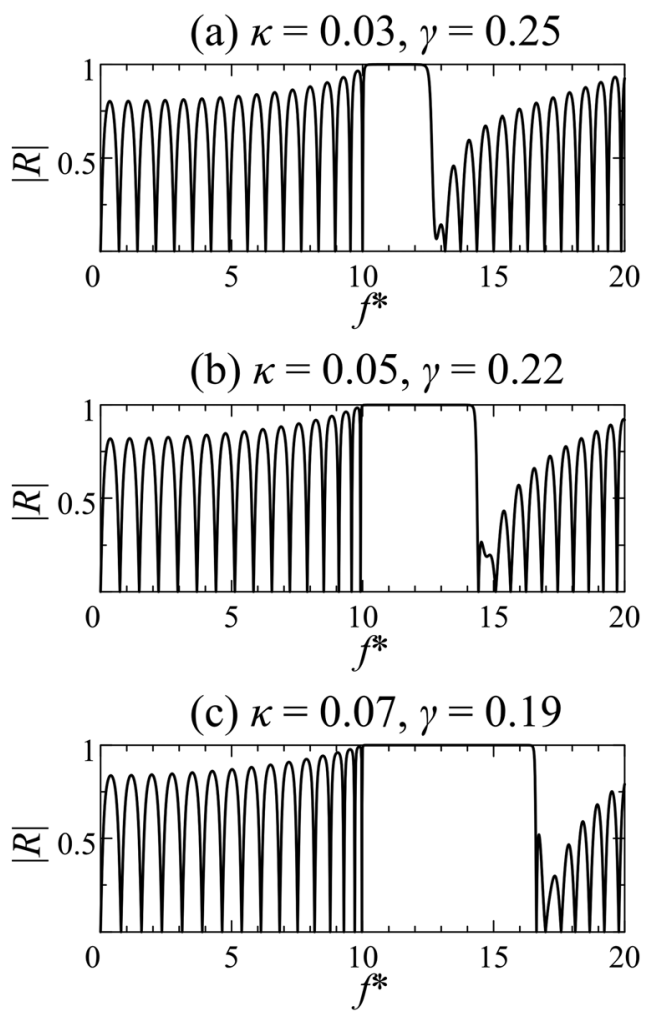

FIG. 6. The amplitude reflection spectra of a multilayered structure for $N=16$ and $\chi=0.84$, (a) $\kappa=0.03$ and $\gamma=0.25$, (b) $\kappa=0.05$ and $\gamma=0.22$, and (c) $\kappa=0.07$ and $\gamma=0.19$.

$$
\varepsilon_{p}=\frac{\varepsilon_{f_{\mathrm{e}}^{*}}}{X_{f_{\mathrm{e}}^{*}, p}},
$$

where $X_{f_{\mathrm{e}}^{*}, p}$ represents the sensitivity of $f_{\mathrm{e}}^{*}$ to $p$, defined as

$$
X_{f_{\mathrm{e}}^{*}, p}=\frac{p}{f_{\mathrm{e}}^{*}} \frac{\partial f_{\mathrm{e}}^{*}}{\partial p} .
$$

Equation (10) shows that the higher the sensitivity is, the smaller the influence of the error in the extremal frequencies on the determination of $p$ is, i.e., $p$ can be identified more robustly. Although the sensitivity in Eq. (11) can be in principle obtained from Eq. (1), it requires cumbersome calculations; hence, the sensitivity of the zero-reflection frequencies of the spectrum $f_{\mathrm{z}}^{*}$, i.e., $X_{f_{\mathrm{z}}^{*}, p}$, is considered here, which is easier to obtain from Eq. (1d). Their explicit expressions are given in the Appendix.

The sensitivity of $f_{\mathrm{z}}^{*}$ to $\kappa$ and $\gamma$ is illustrated in Figs. 7 and 8 , respectively, for various $\kappa$ and $\gamma$ with fixed $N=16$ and $\chi=0.84$. Note that although only the zero-reflection frequencies are dealt with here, it is reasonable to expect that the sensitivity of the other extremal frequencies (local maxima and minima) of the reflection spectrum is more or less on the solid lines drawn in the figures. It is found in Figs. 7 and 8 that as $\kappa$ increases or $\gamma$ decreases, the sensitivity to $\kappa$ becomes higher and that to $\gamma$ becomes smaller, indicating that the larger $\kappa(\gamma)$ and the smaller $\gamma(\kappa)$ make the evaluation of $\kappa(\gamma)$ more sensitive and that of $\gamma(\kappa)$ less sensitive. As an example, the evaluation functions for two cases are depicted in Fig. 9 [Fig. 9(a) is for large $\kappa_{\mathrm{o}}$ and small $\gamma_{\mathrm{o}}$ and Fig. 9(b)
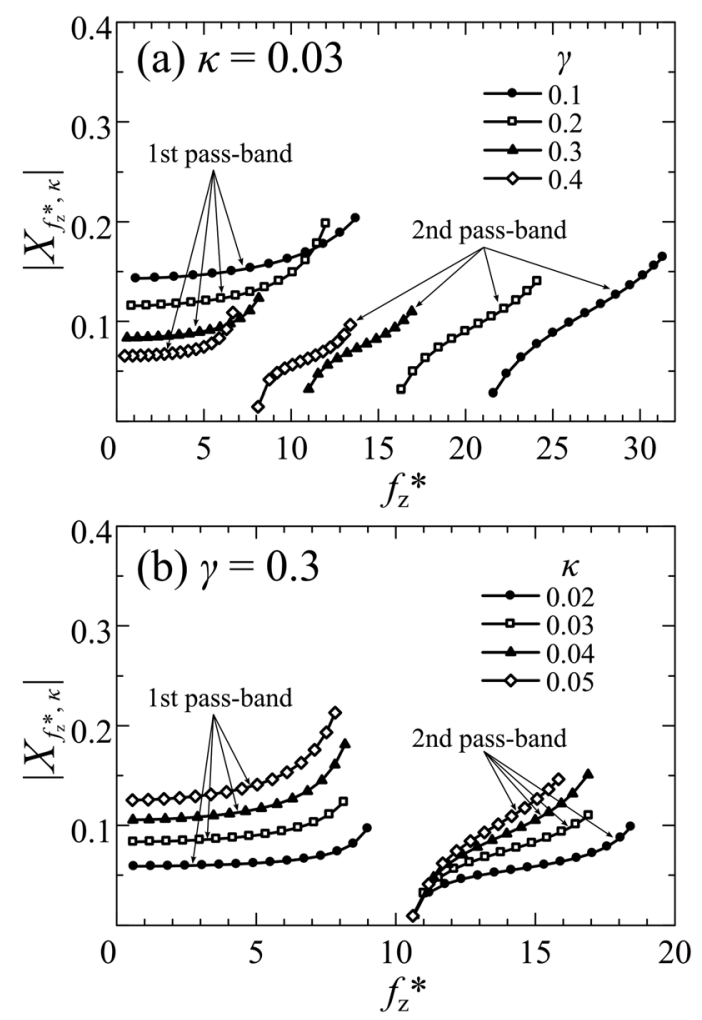

FIG. 7. The sensitivity of the zero-reflection frequencies of the spectrum to $\kappa$ for $N=16$ and $\chi=0.84$, (a) $\gamma=0.1,0.2,0.3$, and 0.4 with a fixed $\kappa=0.03$ and (b) $\kappa=0.02,0.03,0.04$, and 0.05 with a fixed $\gamma=0.3$. 

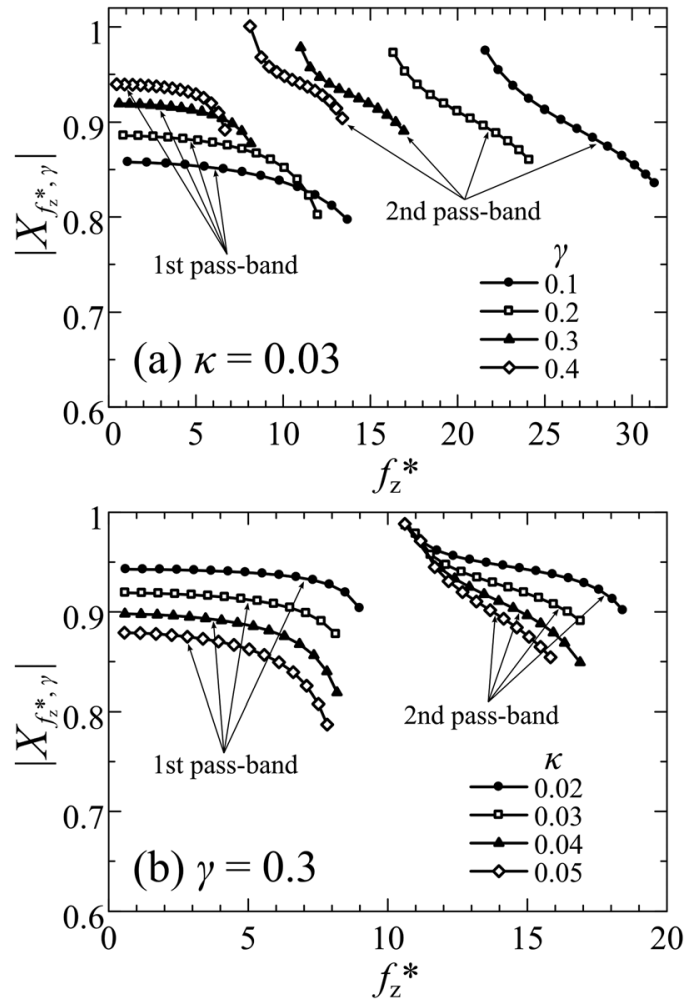

FIG. 8. The sensitivity of the zero-reflection frequencies of the spectrum to $\gamma$ for $N=16$ and $\chi=0.84$, (a) $\gamma=0.1,0.2,0.3$, and 0.4 with a fixed $\kappa=0.03$ and (b) $\kappa=0.02,0.03,0.04$, and 0.05 with a fixed $\gamma=0.3$.
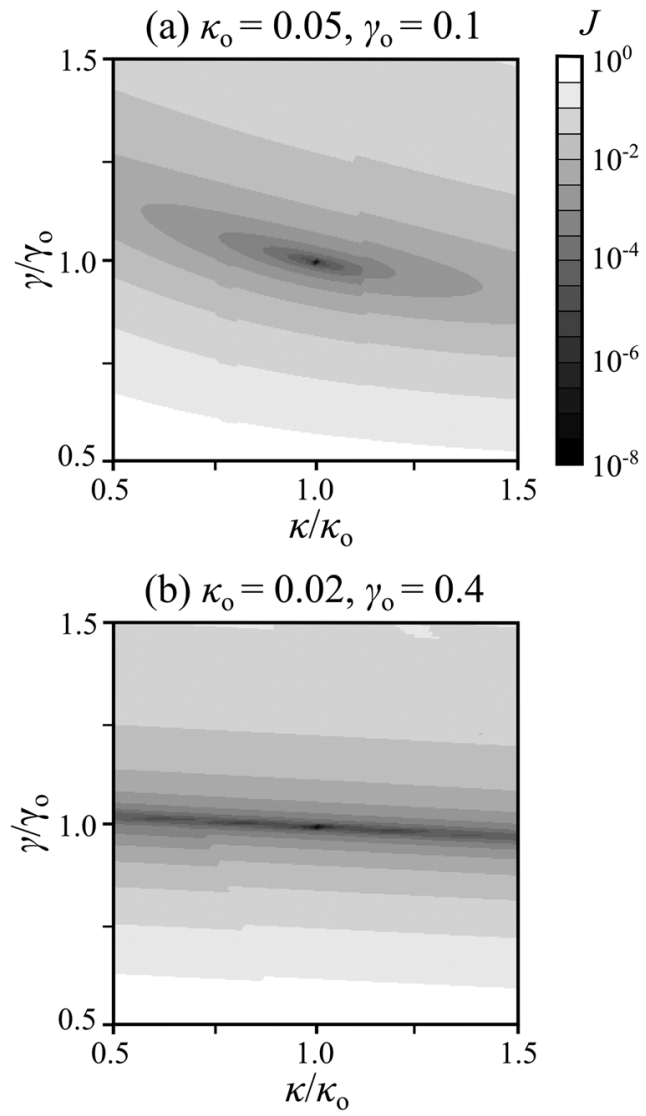

FIG. 9. The evaluation function for fixed $N=16$ and $\chi=0.84$ when the actual non-dimensional parameters are (a) $\kappa_{\mathrm{o}}=0.05$ and $\gamma_{\mathrm{o}}=0.1$ and (b) $\kappa_{\mathrm{o}}=0.02$ and $\gamma_{\mathrm{o}}=0.4$. for small $\kappa_{\mathrm{o}}$ and large $\gamma_{\mathrm{o}}$, where the subscript "o" denotes the actual value]. It is recognized that the optimal $\kappa$ in Fig. 9(a) is determined more robustly due to its higher sensitivity than that in Fig. 9(b). Furthermore, in spite of the lower sensitivity to $\gamma$ than that in Fig. 9(b), the optimal $\gamma$ in Fig. 9(a) is still determined with relatively high robustness. This is because even if the sensitivity to $\gamma$ decreases, its magnitude is still sufficiently large in comparison with that to $\kappa$ (see the vertical axes of Figs. 7 and 8).

The proposed procedure is consequently capable of evaluating $\kappa$ and $\gamma$ sensitively, except for the small $\kappa$ range. However, small $\kappa$, i.e., high interlayer interfacial stiffness $K_{\mathrm{N}}$, implies that the interlayer interface can to a good approximation be considered as a perfectly bonded interface; therefore precise evaluation of $\kappa$ may be unnecessary.

\section{Influence of fluctuations of interlayer interfacial stiffness}

It is expected that the material properties of actual multilayered structures, in particular the interlayer interfacial stiffness, are not identical for the whole structure but have some statistical fluctuations. Even in this situation, it is desirable that the proposed procedure gives some kind of representative interfacial stiffness. The performance of the proposed procedure in such situations is examined below. It should be noted that this study deals with the case where all interfaces still have close stiffnesses in the presence of fluctuations; namely, the case where, for example, a specific interface has a greatly different stiffness from the others is not considered here.

To this aim, the amplitude reflection spectrum for a multilayered structure with random fluctuations of the interlayer interfacial stiffness $K_{\mathrm{N}}$ is calculated by the transfer-matrix method. Figure 10(b) shows the computed reflection spectrum for one sample of the fluctuated $\kappa$ shown in Fig. 10(a) with fixed $N=16, \gamma=0.283$, and $\chi=0.84$, where the value of $K_{\mathrm{N}}$ for each interlayer interface follows the Gaussian distribution with the mean value $\overline{K_{\mathrm{N}}}=0.30 \mathrm{MPa} / \mathrm{nm}$ and the standard deviation $\sigma_{K}=0.05 \mathrm{MPa} / \mathrm{nm}$, and $\kappa$ is calculated by using the properties shown in Table I and the normalization factor $f_{0}=1.00 \mathrm{MHz}$. The reflection spectrum computed without the fluctuation $\left(K_{\mathrm{N}}=0.30 \mathrm{MPa} / \mathrm{nm}\right.$ for all interfaces $)$ is also shown in Fig. 10(b) for comparison. It is seen in Fig. 10(b) that due to the introduced fluctuation, the magnitude of the reflection coefficient is remarkably disturbed from the one without fluctuation; nevertheless, the presence of the extremal points can be observed. Using these points, the evaluation function is computed and illustrated in Fig. 11, where the vertical and horizontal axes are normalized by $\kappa_{\mathrm{o}}=4.68 \times 10^{-2}$ and $\gamma_{\mathrm{o}}=0.283$ (corresponding to $\overline{K_{\mathrm{N}}}$ $=0.30 \mathrm{MPa} / \mathrm{nm}$ and $c=3.00 \mathrm{~km} / \mathrm{s}$ ), respectively. It is found that although the fluctuation makes the optimum unclear in comparison with the case without fluctuation in Fig. 11(b), the minimum point can be still obtained at $\kappa / \kappa_{\mathrm{o}}=1.10$ and $\gamma / \gamma_{\mathrm{o}}=1.01$ (corresponding to $K_{\mathrm{N}}=0.27 \mathrm{MPa} / \mathrm{nm}$ and $c=2.97 \mathrm{~km} / \mathrm{s}$ ) in Fig. 11(a).

The above procedure is repeated for 1000 different distributions of $K_{\mathrm{N}}$ generated randomly for two types of 
(a) Distribution of $K_{\mathrm{N}}$

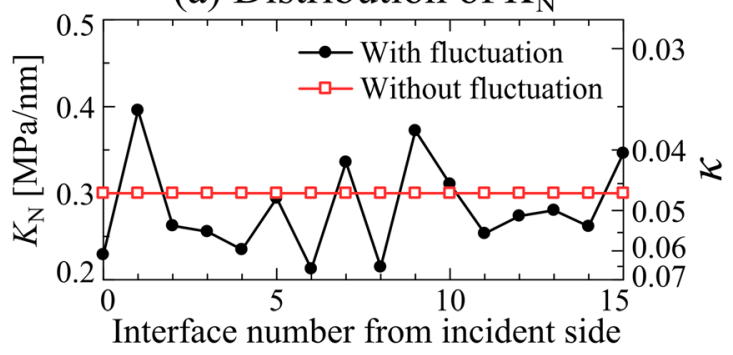

(b) Reflection spectrum

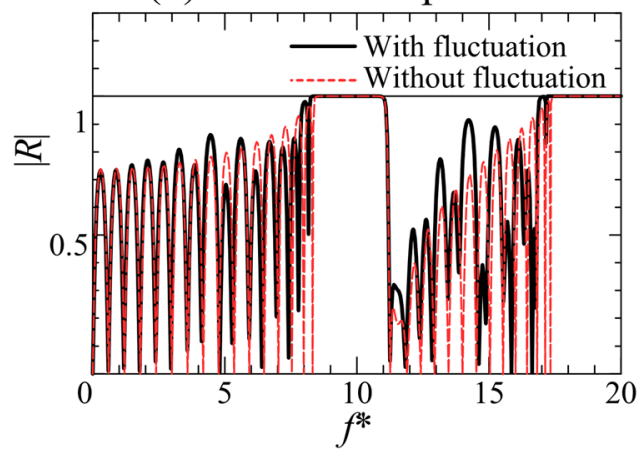

FIG. 10. (Color online) (a) The distribution of the fluctuated interlayer interfacial stiffness $K_{\mathrm{N}}(\kappa)$ through the stacking direction and (b) the computed amplitude reflection spectrum with fixed $N=16, \gamma=0.283$, and $\chi=0.84$, together with the one without fluctuation of $K_{\mathrm{N}}$.

standard deviations (one is for $\sigma_{K}=0.01 \mathrm{MPa} / \mathrm{nm}$ and the other for $\left.\sigma_{K}=0.05 \mathrm{MPa} / \mathrm{nm}\right)$ with fixed $\overline{K_{\mathrm{N}}}=0.30 \mathrm{MPa} / \mathrm{nm}$, $N=16, \gamma=0.283$, and $\chi=0.84$. The optimum point at each trial is searched by using the Fletcher-Reeves conjugate gradient method ${ }^{34}$ with the initial guess $\kappa / \kappa_{\mathrm{o}}=\gamma / \gamma_{\mathrm{o}}=0.9$. It is noted that in Fig. 11(a) there are no local minima other than the global minimum (optimum), implying that the minimization process converges to the optimum as long as the initial guess is chosen within the range shown in the figure. The evaluated results are shown as histograms in Fig. 12. It is found in Fig. 12 that although the dispersion of the identified results is dependent on $\sigma_{K}$, the average interfacial stiffness as well as the originally fixed wave velocity in the layers can be obtained with reasonable accuracy by the present evaluation procedure.

\section{APPLICATION TO CARBON-FIBER-REINFORCED COMPOSITE LAMINATE}

In the previous study, ${ }^{25}$ the interlayer interfacial stiffness of a 16-layered cross-ply laminate of carbon-fiber-reinforced epoxy composite was evaluated with the assumption that all of the acoustic properties of plies were known in advance. In the present study, for the same sample, the ply

TABLE I. Material properties of the layer and the surrounding medium (water).

\begin{tabular}{lc}
\hline \hline Density of layer $\rho$ & $1.49 \times 10^{3} \mathrm{~kg} / \mathrm{m}^{3}$ \\
Thickness of layer $h$ & $0.135 \mathrm{~mm}$ \\
Density of surrounding medium $\rho_{0}$ & $0.998 \times 10^{3} \mathrm{~kg} / \mathrm{m}^{3}$ \\
Longitudinal wave velocity in surrounding medium $c_{0}$ & $1.50 \mathrm{~km} / \mathrm{s}$ \\
\hline
\end{tabular}

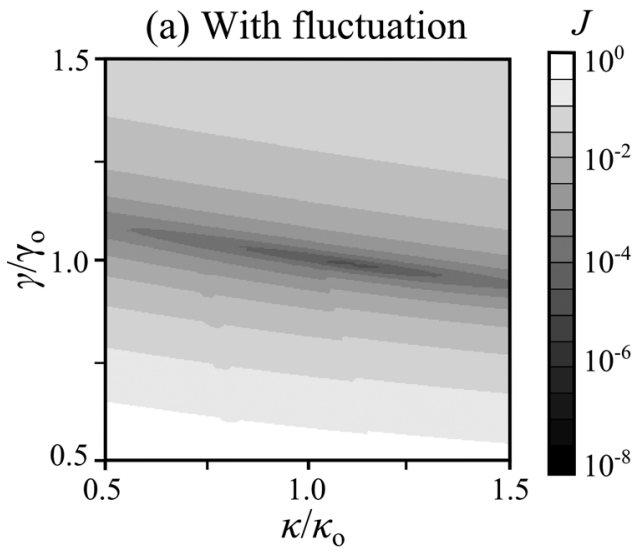

(b) Without fluctuation

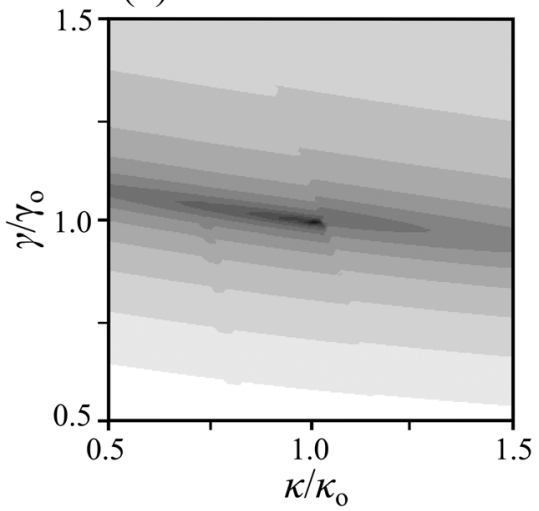

FIG. 11. The evaluation function computed for the spectra in Fig. 10(b), where the vertical and horizontal axes are respectively normalized by $\kappa_{\mathrm{o}}=4.68 \times 10^{-2}$ and $\gamma_{\mathrm{o}}=0.283$ (corresponding to $\overline{K_{\mathrm{N}}}=0.30 \mathrm{MPa} / \mathrm{nm}$ and $c=3.00 \mathrm{~km} / \mathrm{s}$ ).

longitudinal wave velocity $c$ is assumed to be unknown and evaluated simultaneously with the interlayer interfacial normal stiffness $K_{\mathrm{N}}$, where the other parameters are assumed to be known. These known parameters are given as in Table I and the normalization factor $f_{0}$ is set as $1.00 \mathrm{MHz}$, which correspond to the non-dimensional parameter $\chi=0.84$.

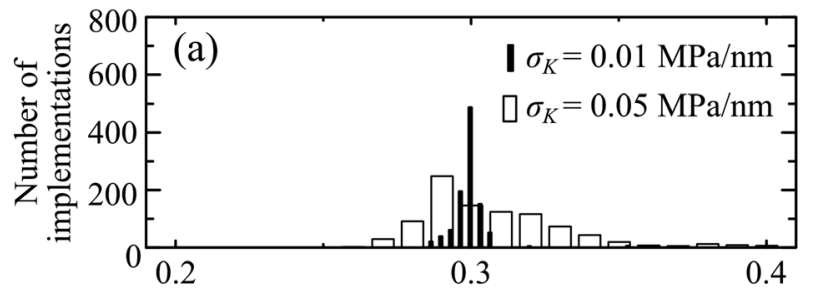

Evaluated interlayer interfacial stiffness $K_{\mathrm{N}}[\mathrm{MPa} / \mathrm{nm}]$

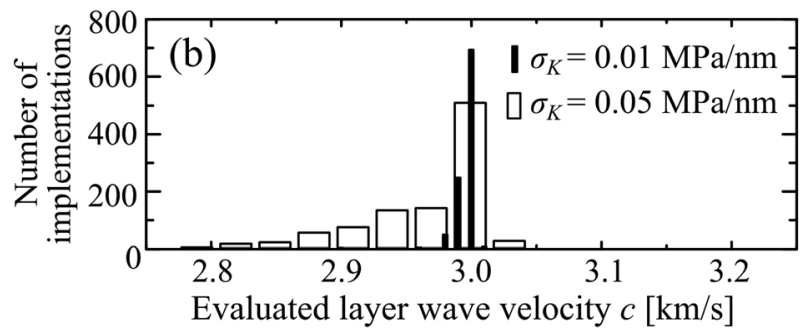

FIG. 12. The histogram of the evaluated (a) $K_{\mathrm{N}}$ and (b) $c$ for two different standard deviations of the fluctuation of $K_{\mathrm{N}}$ with fixed $\overline{K_{\mathrm{N}}}=0.30 \mathrm{MPa} / \mathrm{nm}$ and $c=3.00 \mathrm{~km} / \mathrm{s}$. 
It is noted that the inhomogeneity scale of the unidirectional carbon-fiber-reinforced composites, i.e., the fiber diameter and the interval of the fiber placement, is much smaller than the wavelength of the practically used ultrasonic waves. In this case, each ply can be modeled as a homogeneous layer, and each resin-rich region between neighboring plies as a spring interface. Furthermore, even if the fiber directions of unidirectionally reinforced plies are different such as in angle-ply, cross-ply, or quasi-isotropic laminates, the acoustic properties in the stacking direction are still identical for all plies. Therefore insofar as the normal incidence of the longitudinal wave is considered, the composite laminate can be treated as the multilayered structure considered in Sec. II.

The experimental reflection spectrum of the abovementioned composite laminate is shown in Fig. 13 (see Ref. 25 for the procedure to obtain the spectrum). Although the spectrum is only shown for a finite frequency range from 5 to $15 \mathrm{MHz}$ due to the limited bandwidth of the measurement, it contains local maxima and minima in the first as well as second passbands (a comparatively high peak at around 10.5 MHz corresponds to the first stop band), which is sufficient for unique determination of $K_{\mathrm{N}}$ and $c$ (Sec. III B 2). Using 27 extremal points shown in Fig. 13, the evaluation function Eq. (9) is calculated and shown in Fig. 14. It is found in Fig. 14 that the optimal point can be obtained at $\kappa=1.10 \times 10^{-2}$ and $\gamma=0.275$. Substituting these values into Eq. (8), the interlayer interfacial stiffness and the ply wave velocity of the composite laminate are finally identified as $K_{\mathrm{N}}=1.3 \mathrm{MPa} / \mathrm{nm}$ and $c=3.08 \mathrm{~km} / \mathrm{s}$. The evaluated interfacial stiffness appears reasonable in comparison to a rough estimation ${ }^{25}$ of $K_{\mathrm{N}}$ based on the micrographic observation of the sample and a simple relation ${ }^{12} K_{\mathrm{N}}=\left(\lambda_{\mathrm{e}}+2 \mu_{\mathrm{e}}\right) / h_{\mathrm{e}}$ $\left(\lambda_{\mathrm{e}}+2 \mu_{\mathrm{e}}=8.8 \mathrm{GPa}\right.$ : elastic constants of epoxy and $h_{\mathrm{e}}=3$ to $8 \mu \mathrm{m}$ : thickness of resin-rich zone), which gives a range of $K_{\mathrm{N}}$ as 1.1 to $2.9 \mathrm{MPa} / \mathrm{nm}$. The evaluated wave velocity is also reasonable for unidirectional carbon-fiber-reinforced plies, which indicates that the present method is applicable for the situation where the layer wave velocity is unknown beforehand, as long as all plies have almost the same velocity. Furthermore, the ply stiffness in the direction normal to

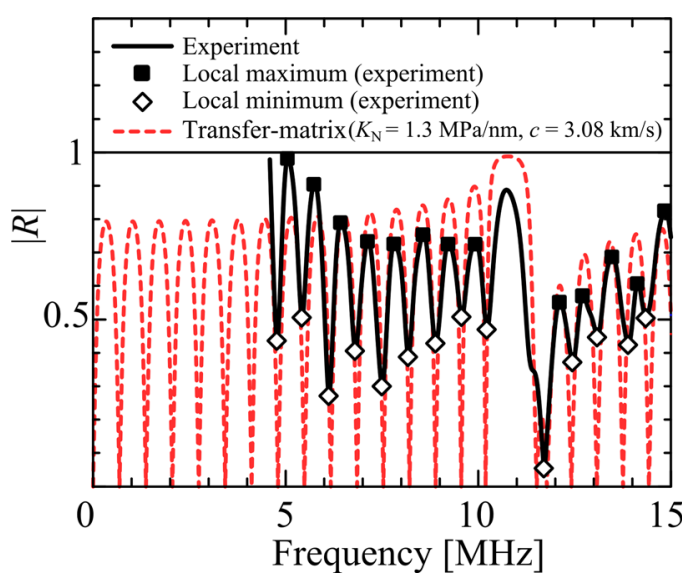

FIG. 13. (Color online) Experimental and theoretical amplitude reflection spectra of the 16-layered composite laminate.

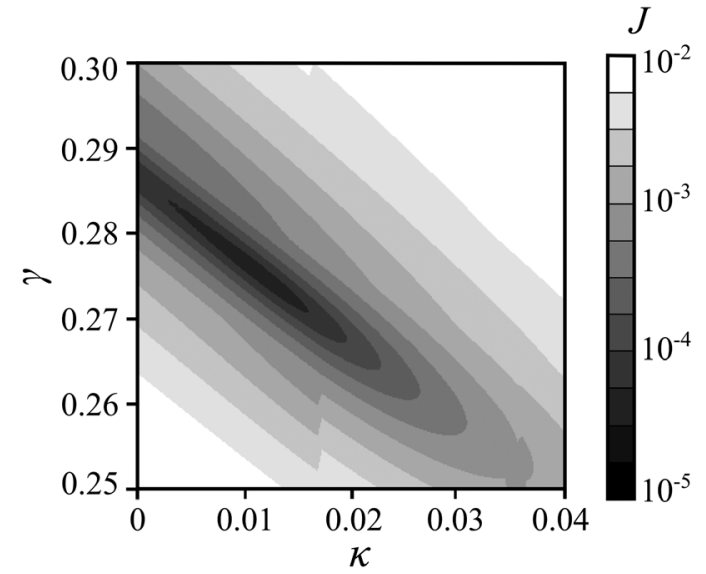

FIG. 14. The experimental evaluation function, where the optimal point is obtained at $\kappa=1.10 \times 10^{-2}$ and $\gamma=0.275$ (corresponding to $K_{\mathrm{N}}$ $=1.3 \mathrm{MPa} / \mathrm{nm}$ and $c=3.08 \mathrm{~km} / \mathrm{s}$ ).

the plies is obtained as $D=\rho c^{2}=14.1 \mathrm{GPa}$ which compares favorably with an existing theoretical result. ${ }^{35}$

The obtained interfacial stiffness is slightly different from the value $K_{\mathrm{N}}=1.6 \mathrm{MPa} / \mathrm{nm}$ determined with the $a \mathrm{pri}$ ori assumed ply wave velocity $c=3.06 \mathrm{~km} / \mathrm{s}$ in the previous study. ${ }^{25}$ Since it has been shown ${ }^{25}$ that the evaluated value of $K_{\mathrm{N}}$ is quite sensitive to the assumed value of $c$, the present procedure is advantageous in that $K_{\mathrm{N}}$ and $c$ can be evaluated simultaneously. Using the so-obtained $K_{\mathrm{N}}$ and $c$, the theoretical amplitude reflection spectrum is computed by the transfer-matrix method and shown in Fig. 13 together with the experimental result. Although the magnitude of the reflection coefficient of the experimental result does not agree with the theoretical one due mainly to the viscoelastic nature of the composite laminate sample, the oscillatory characteristics against the frequency of the experimental result are well reproduced by the theory.

\section{CONCLUSION}

Based on the characteristics of the amplitude reflection spectrum for a multilayered plate-like structure with springtype interlayer interfaces, a procedure to identify the interlayer interfacial normal stiffness as well as the layer longitudinal wave velocity from local maximum and minimum frequencies of the reflection spectrum has been proposed. The influence of the number of extrema to be used in the evaluation has been discussed and it has been shown that in addition to extremal frequencies in the first passband, those in the second passband have to be included for unique determination of the interfacial stiffness and the layer wave velocity. The effectiveness of the proposed procedure has been discussed from the perspective of the sensitivity of local extremal frequencies of the amplitude reflection spectrum. The proposed procedure has been shown to be feasible even when the interlayer interfacial stiffness is randomly fluctuated across the stacking direction. Using the proposed procedure, the interlayer interfacial normal stiffness and the ply longitudinal wave velocity of a carbon-fiber-reinforced composite laminate have been evaluated from the experimental reflection spectrum. 
In this study, the evaluation has been performed for an undamaged composite laminate. Applications to damaged samples as well as the evaluation of other parameters such as interfacial shear stiffness are left for the future study.

\section{ACKNOWLEDGMENTS}

This work has been supported by JSPS KAKENHI Grant Numbers 25-1754 and 25289005.

\section{APPENDIX: EXPRESSSION OF SENSITIVITY OF ZERO- REFLECTION FREQUENCIES}

From Eqs. (1d), (2b), and (7), the zero-reflection frequencies of the amplitude reflection $\operatorname{spectrum} f_{\mathrm{z}}^{*}$ satisfy

$$
\begin{aligned}
& C_{\beta}=C_{\gamma f_{\mathrm{z}}^{*}}-\kappa f_{\mathrm{z}}^{*} S_{\gamma f_{\mathrm{z}}^{*}}, \\
& 2 \kappa \gamma^{2} f_{\mathrm{z}}^{*} S_{(N-1) \beta}-\left(\chi^{2}-\gamma^{2}\right) S_{N \beta} S_{\gamma f_{\mathrm{z}}^{*}}=0,
\end{aligned}
$$

where $S_{x} \equiv \sin x$ and $C_{x} \equiv \cos x$. The differentiation of the above equations with respect to $\kappa$ and $\gamma$ respectively leads to the expressions of the sensitivity as

$$
\begin{aligned}
& X_{f_{\mathrm{z}}^{*}, \kappa}=\frac{\kappa}{f_{\mathrm{z}}^{*}} \frac{\partial f_{\mathrm{z}}^{*}}{\partial \kappa}=\frac{\Lambda_{4}}{\Lambda_{1} \Lambda_{2}+\Lambda_{3}}, \\
& X_{f_{\mathrm{z}}^{*}, \gamma}=\frac{\gamma}{f_{\mathrm{z}}^{*}} \frac{\partial f_{\mathrm{z}}^{*}}{\partial \gamma}=\frac{\Lambda_{5}}{\Lambda_{1} \Lambda_{2}+\Lambda_{3}},
\end{aligned}
$$

where

$$
\Lambda_{1}=2 \kappa \gamma^{2} f_{\mathrm{Z}}^{*}(N-1) C_{(N-1) \beta}-N\left(\chi^{2}-\gamma^{2}\right) C_{N \beta} S_{\gamma f_{\mathrm{z}}^{*}},
$$

$$
\Lambda_{2}=f_{\mathrm{z}}^{*}\left\{(\gamma+\kappa) S_{\gamma f_{\mathrm{z}}^{*}}+\kappa \gamma f_{\mathrm{z}}^{*} C_{\gamma f_{\mathrm{z}}^{*}}\right\}
$$

$$
\Lambda_{3}=\left(\chi^{2}-\gamma^{2}\right)\left(S_{\gamma f_{z}^{*}}-\gamma f_{z}^{*} C_{\gamma f_{z}^{*}}\right) S_{\beta} S_{N \beta},
$$$$
\Lambda_{4}=-\kappa f_{\mathrm{z}}^{*}\left(2 \gamma^{2} S_{\beta} S_{(N-1) \beta}+\Lambda_{1} S_{\gamma f_{z}^{*}}\right),
$$

$$
\begin{aligned}
\Lambda_{5}= & \left\{\left(\chi^{2}-\gamma^{2}\right) \gamma f_{\mathrm{Z}}^{*} C_{\gamma f_{\mathrm{z}}^{*}}-2 \chi^{2} S_{\gamma f_{\mathrm{z}}^{*}}\right\} S_{\beta} S_{N \beta} \\
& -\gamma f_{\mathrm{Z}}^{*}\left(S_{\gamma f_{\mathrm{z}}^{*}}+\kappa f_{\mathrm{Z}}^{*} C_{\gamma f_{\mathrm{z}}^{*}}\right) \Lambda_{1} .
\end{aligned}
$$

${ }^{1}$ W. M. Ewing, W. S. Jardetzky, and F. Press, Elastic Waves in Layered Media (McGraw-Hill, New York, 1957), 380 pp.

${ }^{2}$ L. M. Brekhovskikh, Waves in Layered Media (Academic, New York, 1960), $561 \mathrm{pp}$.

${ }^{3}$ M. J. S. Lowe, "Matrix techniques for modeling ultrasonic waves in multilayered media," IEEE Trans. Ultrason. Ferroelec. Freq. Control 42(4), 525-542 (1995).

${ }^{4}$ S. I. Rokhlin, "Recent advances in waves in layered media," J. Phys. IV(2), 819-826 (1992).

${ }^{5}$ A. Boström and G. Wickham, "On the boundary conditions for ultrasonic transmission by partially closed cracks," J. Nondestruct. Eval. 10(4), 139-149 (1991).

${ }^{6}$ S. I. Rokhlin and Y. J. Wang, "Analysis of boundary conditions for elastic wave interaction with an interface between two solids," J. Acoust. Soc. Am. 89(2), 503-515 (1991).

${ }^{7}$ S. I. Rokhlin and Y. J. Wang, "Equivalent boundary conditions for thin orthotropic layer between two solids: Reflection, refraction, and interface waves," J. Acoust. Soc. Am. 91(4), 1875-1887 (1992).

${ }^{8}$ S. I. Rokhlin and W. Huang, "Ultrasonic wave interaction with a thin anisotropic layer between two anisotropic solids: Exact and asymptotic-boundary condition methods," J. Acoust. Soc. Am. 92(3), 1729-1742 (1992).
${ }^{9}$ S. I. Rokhlin and W. Huang, "Ultrasonic wave interaction with a thin anisotropic layer between two anisotropic solids. II. Second-order asymptotic boundary conditions," J. Acoust. Soc. Am. 94(6), 3405-3420 (1993).

${ }^{10} \mathrm{~A}$. Boström and M. Golub, "Elastic SH wave propagation in a layered anisotropic plane with interface damage modelled by spring boundary conditions," Q. J. Mech. Appl. Math. 62(1), 39-52 (2009).

${ }^{11}$ M. V. Golub, "Propagation of elastic waves in layered composites with microdefect concentration zones and their simulation with spring boundary conditions," Acoust. Phys. 56(6), 848-855 (2010).

${ }^{12}$ A. Pilarski and J. L. Rose, "A transverse-wave ultrasonic obliqueincidence technique for interfacial weakness detection in adhesive bonds," J. Appl. Phys. 63(2), 300-307 (1988).

${ }^{13}$ P. Fraisse, F. Scmit, and A. Zarembowitch, "Ultrasonic inspection of very thin adhesive layers," J. Appl. Phys. 72(8), 3264-3271 (1992).

${ }^{14}$ P. B. Nagy, "Ultrasonic classification of imperfect interfaces," J. Nondestruct. Eval. 11, 127-139 (1992).

${ }^{15}$ B. W. Drinkwater, R. S. Dwyer-Joyce, and P. Cawley, "A study of the transmission of ultrasound across solid-rubber interfaces," J. Acoust. Soc. Am. 101(2), 970-981 (1997).

${ }^{16} \mathrm{~S}$. Rokhlin, M. Hefets, and M. Rosen, "An elastic interface wave guided by a thin film between two solids," J. Appl. Phys. 51(7), 3579-3582 (1980).

${ }^{17}$ B. Drinkwater, M. Castaings, and B. Hosten, "The measurement of $\mathrm{A}_{0}$ and $\mathrm{S}_{0}$ Lamb wave attenuation to determine the normal and shear stiffness of a compressively loaded interface," J. Acoust. Soc. Am. 113(6), 3161-3170 (2003).

${ }^{18}$ S. Biwa, S. Hiraiwa, and E. Matsumoto, "Stiffness evaluation of contacting surfaces by bulk and interface waves," Ultrasonics 47, 123-129 (2007).

${ }^{19}$ N. Mori, S. Biwa, and T. Hayashi, "Reflection and transmission of Lamb waves at an imperfect joint of plates," J. Appl. Phys. 113, 074901 (2013).

${ }^{20}$ A. I. Lavrentyev and S. I. Rokhlin, "Ultrasonic spectroscopy of imperfect contact interfaces between a layer and two solids," J. Acoust. Soc. Am. 103(2), 657-664 (1998).

${ }^{21}$ L. Singher, Y. Segal, E. Segal, and J. Shamir, "Considerations in bond strength evaluation by ultrasonic guided waves," J. Acoust. Soc. Am. 96(4), 2497-2505 (1994).

${ }^{22}$ R. Leiderman, A. M. B. Braga, and P. E. Barbone, "Scattering of ultrasonic waves by defective adhesion interfaces in submerged laminated plates," J. Acoust. Soc. Am. 118(4), 2154-2166 (2005).

${ }^{23}$ R. Leiderman, P. E. Barbone, and A. M. B. Braga, "Reconstructing the adhesion stiffness distribution in a laminated elastic plate: Exact and approximate inverse scattering solutions," J. Acoust. Soc. Am. 122(4), 1906-1916 (2007).

${ }^{24}$ X. M. Jian, N. Guo, M. X. Li, and H. L. Zhang, "Characterization of bonding quality in a multilayer structure using segment adaptive filtering," J. Nondestruct. Eval. 21(2), 55-65 (2002).

${ }^{25}$ Y. Ishii and S. Biwa, "Ultrasonic evaluation of interlayer interfacial stiffness of multilayered structures," J. Appl. Phys. 111, 084907 (2012).

${ }^{26}$ A. Baltazar, L. Wang, B. Xie, and S. I. Rokhlin, "Inverse ultrasonic determination of imperfect interfaces and bulk properties of a layer between two solids," J. Acoust. Soc. Am. 114(3), 1424-1434 (2003).

${ }^{27} \mathrm{~W}$. T. Thomson, "Transmission of elastic waves through a stratified solid medium," J. Appl. Phys. 21, 89-93 (1950).

${ }^{28}$ N. A. Haskell, "The dispersion of surface waves on multilayered media," Bull. Seismol. Soc. Am. 43, 17-34 (1953).

${ }^{29}$ Y. Lu and J. D. Achenbach, "Effects of random deviations in interface properties on the propagation of ultrasound in thick composites," J. Acoust. Soc. Am. 90(5), 2576-2585 (1991).

${ }^{30} \mathrm{Y}$. Lu, "Effects of random fluctuations in ply mechanical properties on ultrasound propagation in a laminated solid layer," Ultrasonics 30(5), 289-295 (1992).

${ }^{31}$ J. M. Bendickson, J. P. Dowling, and M. Scalora, "Analytic expressions for the electromagnetic mode density in finite, one-dimensional, photonic band-gap structures," Phys. Rev. E 53, 4107-4121 (1996).

${ }^{32}$ F. Kobayashi, S. Biwa, and N. Ohno, "Wave transmission characteristics in periodic media of finite length: Multilayers and fiber arrays," Int. J. Solids Struct. 41, 7361-7375 (2004).

${ }^{33}$ A. I. Lavrentyev and S. I. Rokhlin, "Determination of elastic moduli, density, attenuation, and thickness of a layer using ultrasonic spectroscopy at two angles," J. Acoust. Soc. Am. 102(6), 3467-3477 (1997).

${ }^{34}$ R. Fletcher and C. M. Reeves, "Function minimization by conjugate gradients," Comput. J. 7, 149-154 (1964).

${ }^{35}$ R.-B. Yang and A. K. Mal, "Multiple scattering of elastic waves in a fiberreinforced composite," J. Mech. Phys. Solids 42(12), 1945-1968 (1994). 